\title{
Efficacy of Underwater Endoscopic Mucosal Resection for Nonpedunculated Colorectal Polyps: A Systematic Review and Meta-Analysis
}

\author{
Takeshi Yamashina ${ }^{1,2}$, Noboru Hanaoka ${ }^{1}$, Takeshi Setoyama ${ }^{1}$, Jun Watanabe ${ }^{3}$, Masahiro Banno ${ }^{4,5,6}$,
} Hiroyuki Marusawa ${ }^{1}$

1. Gastroenterology and Hepatology, Osaka Red Cross Hospital, Osaka, JPN 2. Gastroenterology and Hepatology, Kansai Medical University Medical Center, Moriguchi, JPN 3. Division of Gastroenterological, General and Transplant Surgery, Jichi Medical University, Shimotsuke, JPN 4. Department of Systematic Reviewers, Systematic Review Workshop Peer Support Group (SRWS-PSG), Osaka, JPN 5. Department of Psychiatry, Nagoya University Graduate School of Medicine, Nagoya, JPN 6. Department of Psychiatry, Seichiryo Hospital, Nagoya, JPN

Corresponding author: Takeshi Yamashina, take8047@gmail.com

\begin{abstract}
Recently, underwater endoscopic mucosal resection (UEMR) without submucosal injection was introduced as a new replacement for conventional EMR (CEMR) and was reported to be useful for resecting large colonic polyps. Here, we aimed to assess the efficacy and safety of these two methods by a systematic review and meta-analysis.
\end{abstract}

We comprehensively searched multiple databases until July 2021 to identify randomized controlled trials (RCTs) comparing UEMR with CEMR. The primary outcomes were the proportion of R0 resection and mean procedure time, and the secondary outcomes were the proportion of en bloc resection and all adverse events. Three reviewers independently searched for articles, extracted data, and assessed the risk of bias. We evaluated the certainty of evidence using the Grading of Recommendations, Assessment, Development, and Evaluation approach. This study was registered in www.protocols.io (Protocol Integer ID: 40849).

We included six RCTs (1,374 polyps). We judged that a meta-analysis was not available, and the data were summarized narratively for the proportion of R0 resection. Regarding procedure time, UEMR likely resulted in a large reduction (mean difference $=-64.3$ seconds; $95 \%$ confidence interval $(\mathrm{CI})=-122.5$ to -6.0 seconds; $\mathrm{I}^{2}=86 \%$; moderate certainty of evidence). UEMR likely resulted in a large increase in en bloc resection (odds ratio $=1.85 ; 95 \% \mathrm{CI}=1.15$ to $2.98 ; \mathrm{I}^{2}=60 \%$; moderate certainty of evidence). Percentages of adverse events were $0-17 \%$ with CEMR and $0-16 \%$ with UEMR.

In summary, UEMR might have higher efficacy than CEMR in the endoscopic resection of nonpedunculated colorectal polyps, with likely a large reduction in procedure time.

Review began 08/04/2021 Review ended 08/09/2021 Published 08/17/2021

\section{○ Copyright 2021}

Yamashina et al. This is an open access article distributed under the terms of the Creative Commons Attribution License CC-BY 4.0., which permits unrestricted use, distribution, and reproduction in any medium, provided the original author and source are credited.
Categories: Internal Medicine, Gastroenterology

Keywords: underwater emr, conventional endoscopic mucosal resection (cemr), colon cancer prevention, colorectal polyp, systematic review and meta-analysis

\section{Introduction And Background}

Colorectal cancer is the third most common cancer (the third most commonly occurring cancer in men and the second most commonly occurring cancer in women) worldwide, and the second cancer with higher mortality. More than 1.9 million new colorectal cancer cases and 935,000 deaths were estimated to occur in 2020 [1]. Despite the multiple factors involved in colorectal cancer development, a good prognosis can be expected if the disease is detected and treated at an early stage. Endoscopic mucosal resection (EMR), widely used in the prevention of colorectal cancer, is performed by injecting fluid under the colonic mucosa at the site of the lesion to prevent excessive coagulation of the colonic wall caused by resection using electrocautery [2]. However, some proficiency in injecting and snaring is required. In addition, as polyp size increases, en bloc resection becomes difficult, which results in a piecemeal resection that can lead to local recurrence, introducing a further clinical problem [3].

Endoscopic submucosal dissection (ESD) facilitates the excision of large superficial colorectal lesions in an en bloc fashion. Furthermore, ESD can achieve a higher en bloc resection rate than EMR, regardless of tumor size or location [4]. However, technical difficulties and being more time-consuming and costly than EMR prevent the adoption of colorectal ESD as one of the standard endoscopic therapies.

Recently, underwater EMR (UEMR) without submucosal injection, as described by Binmoeller et al., was introduced as a new alternative to conventional EMR (CEMR) and has been reported useful for removing large colonic polyps [5]. In UEMR, the bowel lumen is flooded with water instead of air/ $\mathrm{CO}_{2}$, and the polyp is 
removed with no injection. Compared with CEMR, UEMR makes it easier to snare large colorectal polyps, residual recurrent lesions, and rectal neuroendocrine tumors and has the potential to improve treatment outcomes [6,7]. Randomized controlled trials (RCTs) have reported a significantly higher proportion of R0 resection for UEMR [8] and a significantly shorter procedure time than with CEMR [9].

Although several systematic reviews and meta-analyses of UEMR have already shown efficacy and safety, the superiority of UEMR over CEMR has not been sufficiently examined because it has not been compared with CEMR [10] or has been compared with CEMR in a mixture of cohort studies and RCTs [11-14]. RCTs are the most effective way to scientifically validate new medical interventions. Aside from one study by Tziatzios et al., we are unaware of any other meta-analyses of RCTs [15]. Therefore, we excluded cohort studies and conducted a meta-analysis involving additional RCTs.

\section{Review}

\section{Reporting guidelines}

This systematic review was conducted in accordance with the Preferred Reporting Items for Systematic Reviews and Meta-Analyses (PRISMA) guidelines [16]. The study group also followed the recommendations listed in the Cochrane Handbook (Appendix 1) [17]. We published our review protocol in www.protocols.io [18].

\section{Criteria for considering studies for this review}

Types of Studies

We included RCTs that assessed the efficacy of UEMR compared with CEMR for endoscopic removal of colorectal polyps. We did not apply language or country restrictions and included all published and unpublished articles, abstracts of conferences, and letters. We excluded duplicate publications, quasi-RCTs, cluster-randomized trials, crossover studies, quasi-experimental designs, case reports, and nonhuman studies. We did not exclude studies based on the observation length or year of publication. This study involved men and women aged 18 years or older who underwent UEMR or CEMR for colorectal polyps, and excluded participants with UEMR without submucosal injection, pedunculated lesions based on the Paris endoscopic classification [19]; residual or recurrent lesions after endoscopic resection; and colitis ulcer, Crohn's disease lesions, and familial adenomatous polyposis.

\section{Types of Intervention}

The intervention was UEMR with complete deflation of the colorectal lumen air/ $\mathrm{CO}_{2}$ and total immersion of the polyp in water. Following this, the polyp and the surrounding mucosa were snared and removed with electrocautery. The comparator was CEMR with needle injection of water into the submucosa, followed by entrapment of the mucosal protrusion with a snare and resection using electrocautery.

\section{Types of outcome measures}

Primary Outcomes

The primary outcomes are the proportion of $\mathrm{R} 0$ resection and mean procedure time. The proportion of R0 resection was calculated as the number of $\mathrm{R} 0$ resections divided by the total number of polyps. R0 resection was defined as en bloc resection with a confirmed negative resection margin on histology. En bloc resection was defined as the removal of one polyp section that was assessed endoscopically. The procedure time for the UEMR group was calculated from the beginning of immersion in water through the endoscope until the polyp was completely removed. The procedure time for the CEMR group was calculated from the insertion of the injection needle or injection until the polyp was completely removed.

Secondary Outcomes

The secondary outcomes were the proportion of en bloc resection and adverse events. The proportion of en bloc resection was calculated as the number of en bloc resections divided by the total number of polyps. Definitions of adverse events were set by the original authors.

\section{Search methods to identify studies}

Electronic Searches

We searched the electronic databases, Cochrane Central Register of Controlled Trials (CENTRAL), Medical Literature, Analysis and Retrieval System Online (MEDLINE, Ovid, 1946 to August 2020), Excerpta Medica database (EMBASE, ProQuest, 1974 to August 2020), search portal of the World Health Organization International Clinical Trials Registry Platform (ICTRP), and ClinicalTrials.gov (Appendix 2-6 available at https://data.mendeley.com/datasets/ph9hwfn5v4/1) for ongoing or unpublished trials. We updated the 
electronic searches on July 15, 2021. The keywords used in our search were a combination of "polyps" OR "neoplasms" OR "intestines" OR “colorect*" OR "colon*” OR "bowel” OR "rect*" OR "intestinal mucosa" AND “polyp*” OR “neoplas*” OR “tumour" “adenocarcinom*” OR “cancer*” AND “endoscopic mucosal resection” OR “polypectomy” OR “EMR” OR “endoscopic resection” OR "endoscopic mucosectom*” AND "underwater” OR "under water” AND “randomized controlled trial” OR “controlled clinical trial” OR “randomized” OR "placebo" OR “drug therapy” OR "randomly” OR “trial” OR "groups.” We checked the reference lists of the identified studies, including international guidelines [20-23], the reference lists of eligible studies, and articles citing eligible studies. We consulted the authors of original studies for data not yet published and for supplementary data. If published studies were duplicated, only the latest version, or at least the more complete version, was reviewed.

\section{Data collection and analysis}

Study Selection

Three reviewers (TY, TS, and NH) independently checked titles and abstracts and then assessed eligibility based on the full texts identified in the search. If relevant data could not be found, we contacted the original authors. Disagreements between the reviewers were settled by discussion, and if consensus could not be reached, two additional reviewers (JW and MB) acted as arbiters.

\section{Data Extraction and Management}

Three reviewers independently extracted data from the included studies using a standardized data collection form that was pre-evaluated using 10 randomly selected studies. The form comprised information regarding the study design, study population, patient characteristics (sex, mean age, number of patients undergoing UEMR and CEMR, number of polyps, mean polyp size, polyp location, and histological type of polyp), proportion of R0 resection with UEMR and CEMR, proportion of en bloc resection with UEMR and CEMR, mean procedure time for UEMR and CEMR, and percentage of adverse events (bleeding and perforation). Any disagreements were resolved by discussion, and if this failed, two additional reviewers (JW and MB) acted as arbiters.

Assessment of Risk of Bias in the Included Studies

Three reviewers (TY, TS, and NH) independently assessed the risk of bias with the Risk of Bias 2 tool [24]. Disagreements between the three reviewers were discussed, and if consensus could not be achieved, two additional reviewers (JW and MB) acted as arbiters. If a meta-analysis was not feasible, the data were summarized narratively to rate the certainty in evidence using the Grading of Recommendations, Assessment, Development, and Evaluations (GRADE) approach according to a previous report and the synthesis without meta-analysis (SWiM) guideline [25,26].

Measures of Treatment Effects

We pooled the relative risk ratios and 95\% confidence intervals (CIs) for the variables, R0 resection, and en bloc resection. We pooled the mean differences (MDs) and the 95\% CIs for the continuous variables, UEMR procedure time, and CEMR procedure time. If the included studies used several different scales, we pooled the effect estimates with standard MDs.

Unit of Analysis Issues

For continuous data, only the sample size was reduced; means and standard deviations remained unchanged [24]. For multiple comparisons, we included all intervention groups relevant to this review.

\section{Missing Outcomes}

Intention-to-treat analyses were performed for all dichotomous data, as much as possible. We did not impute missing data for continuous data, in accordance with the recommendation in the Cochrane Handbook [17]. We performed a meta-analysis using the data from the original studies. We asked the original authors for data not presented.

Assessment of Heterogeneity

Statistical heterogeneity was assessed by visually inspecting the forest plots and calculating the I ${ }^{2}$ statistic ( $\mathrm{I}^{2}$ values: 0-40\%, might not be important; 30-60\%, may represent moderate heterogeneity; 50-90\%, may represent substantial heterogeneity; 75-100\%, considerable heterogeneity) [17]. When there was substantial heterogeneity $\left(\mathrm{I}^{2}>50 \%\right)$, we assessed the reason for the heterogeneity. The Cochrane $\mathrm{Chi}^{2}$ test (Q-test) was 
performed for the $\mathrm{I}^{2}$ statistic, and p values of less than 0.10 were defined as statistically significant.

Assessment of Reporting Bias

We searched the clinical trials registry system (ClinicalTrials.gov and ICTRP) and performed an extensive literature search for unpublished trials. Although we planned to evaluate potential publication bias by visual inspection of the funnel plot, we did not conduct this test because we found fewer than 10 trials.

Meta-Analysis

We performed the meta-analysis using Review Manager software (RevMan 5.4) and a random-effects model. Some guidelines recommend ESD rather than EMR for nonpedunculated colorectal polyps larger than $20 \mathrm{~mm}[20,21]$. Therefore, we evaluated the ad hoc subgroup analyses of the lesion size ( $\leqslant 20 \mathrm{~mm}$ or $>20 \mathrm{~mm}$ ) to determine the influence of effect modifiers on the results. And as a difference between the protocol and the review, we were unable to perform other prespecified subgroup analyses and sensitivity analyses for R0 resection and procedure time.

\section{Search results}

We searched a total of 218 studies on August 15, 2020 and updated the electronic searches on July 15, 2021 (Figure 1). We finally included six studies with 1,374 polyps that met our inclusion criteria, 675 of which were removed by CEMR and 699 by UEMR [8,9,27-30]. Two studies included three pedunculated polyps $[8,30]$, so these polyps were excluded from the meta-analysis. However, the summary of the characteristics included these data. Three studies were original articles $[8,9,30]$, and the remaining three were abstracts only [27-29]. Table 1 shows a summary of the characteristics of the included studies. The risk of bias for each study is presented in Figures 2-5. Overall, four studies had a high risk of bias as these showed bias in the outcomes measurement. We included all six studies summarized narratively with the primary outcome of the proportion of R0 resection, and meta-analyses with the primary outcome of procedure time and secondary outcomes $[8,9,27-30]$. We found no unpublished studies. 


\section{Cureus}

Figure 1. Preferred reporting items for systematic reviews and metaanalyses (PRISMA) flow diagram of the literature searchresults.

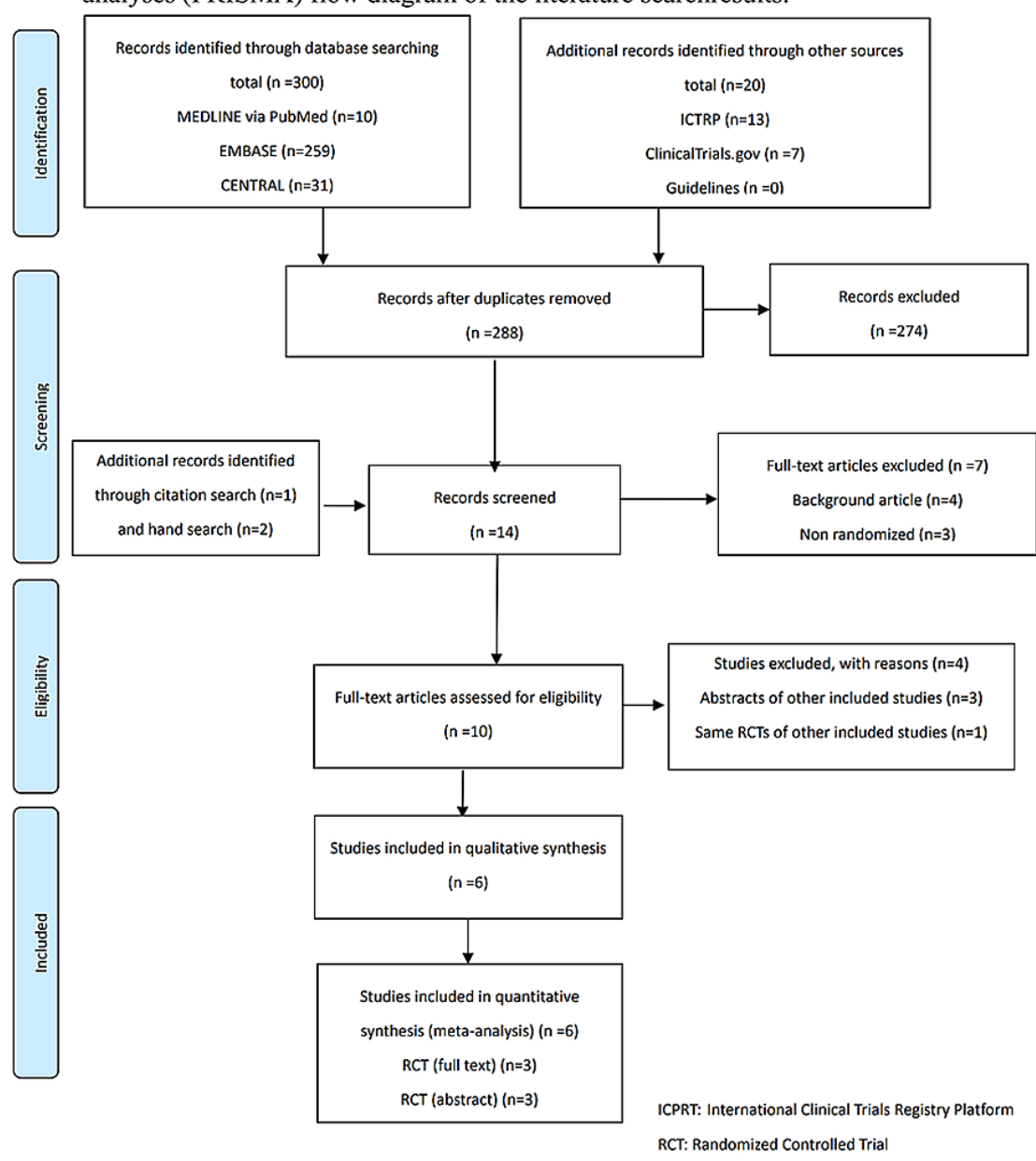

FIGURE 1: PRISMA flow diagram of the literature search results.

PRISMA: Preferred Reporting Items for Systematic Reviews and Meta-Analyses 


\section{Cureus}

\begin{tabular}{|c|c|c|c|c|c|}
\hline Study & Setting & $\begin{array}{l}\text { Enrollment, } \mathrm{n} \\
\text { (patients/polyps) } \\
\text { (male/female) }\end{array}$ & $\begin{array}{l}\text { Number of } \\
\text { polyps } \\
\text { (CEMR/UEMR) }\end{array}$ & $\begin{array}{l}\text { Polyp size } \\
\text { (CEMR/UEMR) }\end{array}$ & $\begin{array}{l}\text { Polyp morphology } \\
\text { (CEMR/UEMR) (\%) }\end{array}$ \\
\hline $\begin{array}{l}\text { Hamerski et } \\
\text { al. (2018) [27] }\end{array}$ & $\begin{array}{l}\text { USA, Multi- } \\
\text { center }\end{array}$ & $178 / 179$ NS & $88 / 91$ & $\begin{array}{l}\text { (mm, mean, range) } 28.1 \\
(15-70) / 29(15-50)\end{array}$ & $\begin{array}{l}\mathrm{Ila} / \mathrm{llb} 69 \mathrm{llc}+\mathrm{lla} / \mathrm{llb} \text { or Ilc } 2 \text { Is+ } \\
\mathrm{Ila} / \mathrm{llb} \text { or Is } 30\end{array}$ \\
\hline $\begin{array}{l}\text { Nagl et al. } \\
\text { (2020) [29] }\end{array}$ & $\begin{array}{l}\text { Germany, } \\
\text { Single- } \\
\text { center }\end{array}$ & NS/117 NS & $59 / 58$ & (mm, range) $20-40^{*}$ & $\begin{array}{l}\text { Large sessile or flat colonic } \\
\text { polyps* }\end{array}$ \\
\hline $\begin{array}{l}\text { Sánchez et } \\
\text { al. (2020) [28] }\end{array}$ & $\begin{array}{l}\text { Spain, } \\
\text { Multi-center }\end{array}$ & NS/267 NS & $141 / 126$ & $32.8^{\dagger}$ & NS \\
\hline $\begin{array}{l}\text { Yamashina et } \\
\text { al. (2019) [8] }\end{array}$ & $\begin{array}{l}\text { Japan, } \\
\text { Multi-center }\end{array}$ & 214/214 139/71 & $102 / 108$ & $\begin{array}{l}\text { (mm, median, range) } \\
13.5(7-25) / 14(7-25)\end{array}$ & $\begin{array}{l}\text { Ila } 57 / 59 \text { Ilc } 0 / 0.9 \text { Is } 43 / 38 \text { Ip } \\
0 / 1.9\end{array}$ \\
\hline $\begin{array}{l}\text { Yen et al. } \\
\text { (2020) [9] }\end{array}$ & $\begin{array}{l}\text { USA, } \\
\text { Single- } \\
\text { center }\end{array}$ & $255 / 462$ 248/7 & $214 / 248$ & $\begin{array}{l}\text { (mm, mean, range) } 9.9 \\
(6-45) / 9.9(6-40)\end{array}$ & $\begin{array}{l}\text { Is } 50.8 / 53.7 \mathrm{lla} 43.2 / 41.1 \mathrm{llb} \\
4.4 / 1.9 \mathrm{llc} 0 / 0.5 \text { Mixed } 0 / 0.5\end{array}$ \\
\hline $\begin{array}{l}\text { Zhang et al. } \\
(2020)[30]\end{array}$ & $\begin{array}{l}\text { China, } \\
\text { Multi-center }\end{array}$ & $130 / 14275 / 55$ & $71 / 71$ & $\begin{array}{l}\text { (mm, median, IQR) } 5.0 \\
(4.0-7.0) / 6.0(5.0-8.0)\end{array}$ & $\begin{array}{l}\text { Is } 70.4 / 76.1 \text { Ip } 0 / 1.4 \text { Ila } \\
29.6 / 22.5\end{array}$ \\
\hline
\end{tabular}

TABLE 1: Summary of the characteristics of the included studies.

NS: not stated; UEMR: underwater endoscopic mucosal resection; CEMR: conventional endoscopic mucosal resection; IQR: interquartile range

*: inclusion criteria, $\uparrow$ : abstract 


\section{Cureus}

\begin{tabular}{|c|c|c|c|c|c|c|}
\hline & $D_{1}$ & D2 & \multicolumn{2}{|c|}{ Risk of bias comains } & D5 & Overall \\
\hline Hamerski 2019 & † & † & † & (8) & † & ( \\
\hline Nagl 2020 & $\odot$ & $\odot$ & $\odot$ & $\odot$ & $\odot$ & $\odot$ \\
\hline Sánchoz 2020 & $\oplus$ & $\oplus$ & $\oplus$ & (2) & $\oplus$ & ( \\
\hline Yamashina 2019 & † & † & † & $\odot$ & $\oplus$ & $\odot$ \\
\hline Yon 2020 & † & $\oplus$ & † & (8) & $\oplus$ & ( \\
\hline Zhang 2020 & † & † & † & Q & † & ( \\
\hline & 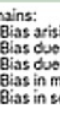 & the repot: & & & & 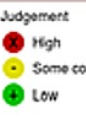 \\
\hline
\end{tabular}

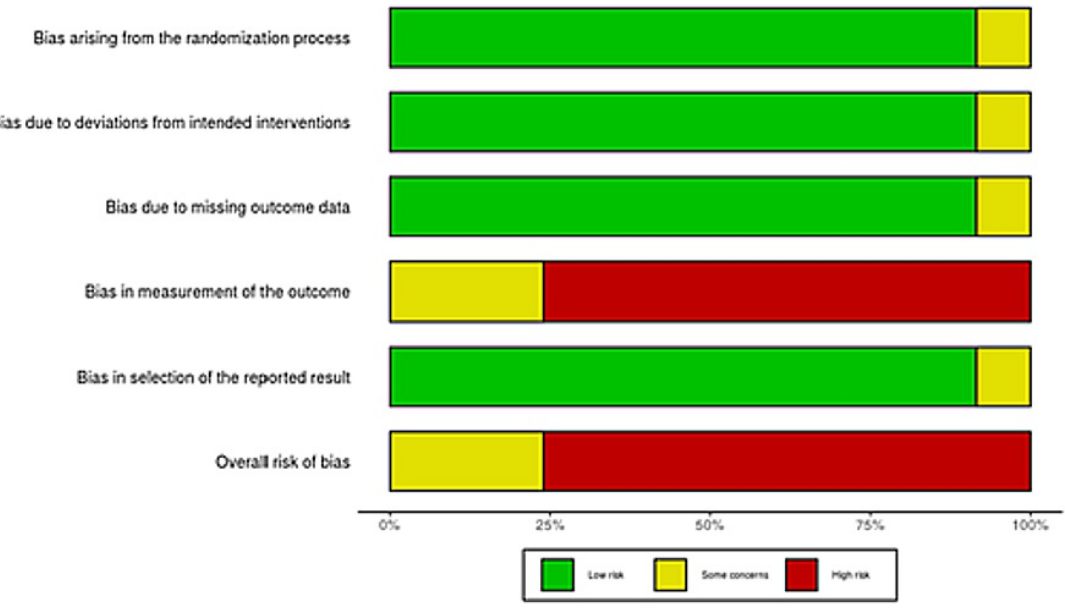

FIGURE 2: Risk-of-bias table and risk-of-bias graph for R0 resection. 


\section{Cureus}

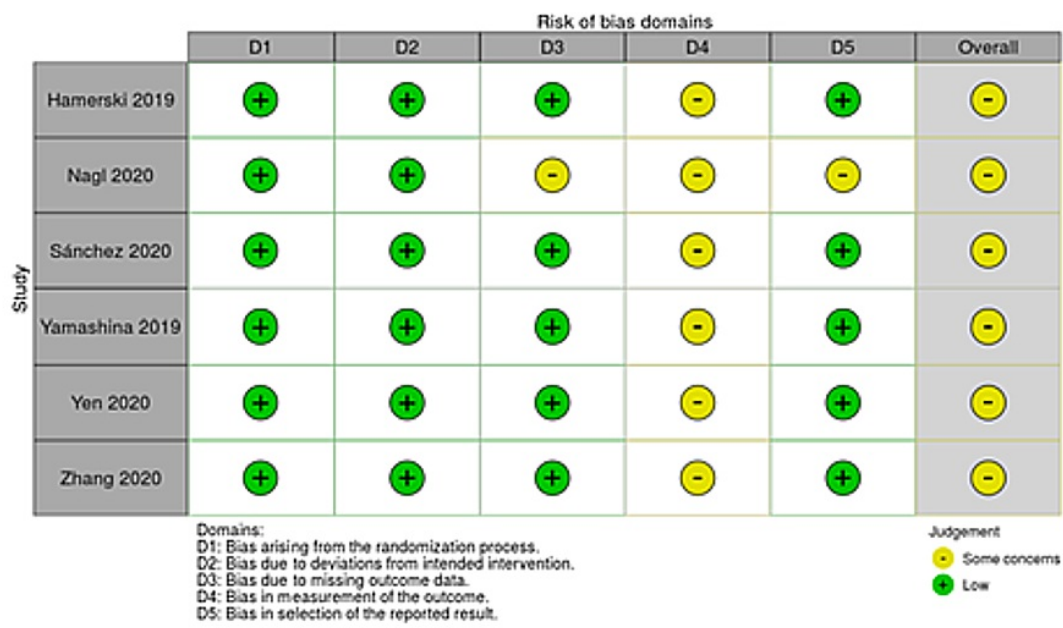

Bias arising from the randomization process

Bias due to deviations trom intended interventions

Biss duo to missing ouscomo but

iss in measurement of the outcome

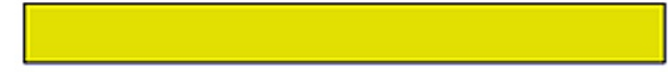

Biss in selection of the reported resull

Overal risk of blas
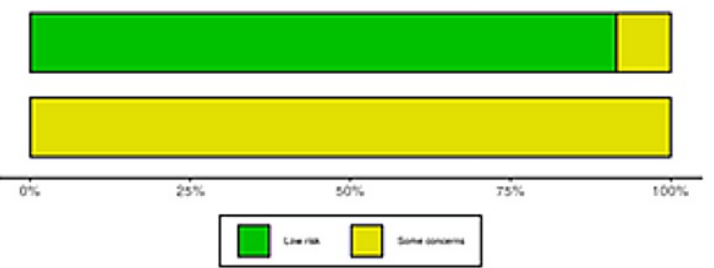

FIGURE 3: Risk-of-bias table and risk-of-bias graph for en bloc resection. 


\section{Cureus}

\begin{tabular}{|c|c|c|c|c|c|c|}
\hline & \multicolumn{6}{|c|}{$\begin{array}{l}\text { Risk of bias domains } \\
\text { D3 }\end{array}$} \\
\hline Hamerski 2019 & † & † & $\oplus$ & $\odot$ & $\oplus$ & $\odot$ \\
\hline Nagl 2020 & † & † & $\odot$ & $\odot$ & $\odot$ & $\odot$ \\
\hline Súnchez 2020 & † & $\oplus$ & $\oplus$ & $\odot$ & $\oplus$ & $\odot$ \\
\hline Yamashina 2019 & † & † & † & $\odot$ & † & $\odot$ \\
\hline Yon 2020 & $\oplus$ & † & $\oplus$ & $\odot$ & $\oplus$ & $\odot$ \\
\hline Zhang 2020 & † & † & $\oplus$ & $\odot$ & $\oplus$ & $\odot$ \\
\hline & 3 & & & & & 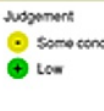 \\
\hline
\end{tabular}

Biss arising frem the randomization process

Biss due to deviations trom intended interventions

Biss duo so missing ounsomo data
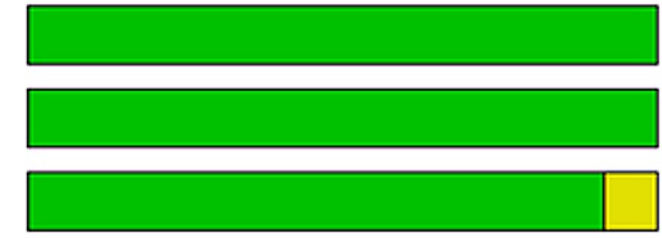

Bias in measurement of the outcome

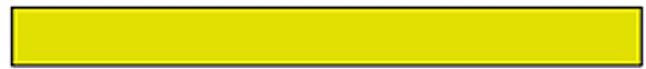

Bas in selection of the teported rewd

Overal risk of blas
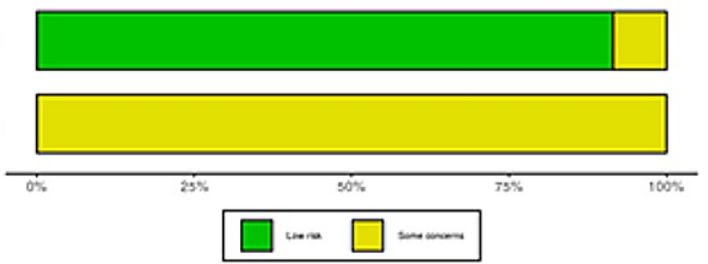

FIGURE 4: Risk-of-bias table and risk-of-bias graph for procedure time. 


\section{Cureus}

\begin{tabular}{|c|c|c|c|c|c|c|}
\hline & \multicolumn{6}{|c|}{ Risk of bias domains } \\
\hline & D1 & D2 & D3 & DA & D5 & Overall \\
\hline Hamerski 2019 & $\oplus$ & $\oplus$ & $\oplus$ & $\odot$ & $\oplus$ & $\bigodot$ \\
\hline Nag1 2020 & $\oplus$ & $\oplus$ & $\odot$ & $\odot$ & $\odot$ & $\bigodot$ \\
\hline Sunchez 2020 & † & † & † & $\odot$ & $\oplus$ & $\bigodot$ \\
\hline Yamashina 2019 & $\oplus$ & $\oplus$ & $\oplus$ & $\odot$ & $\oplus$ & $\odot$ \\
\hline Yon 2020 & $\oplus$ & $\oplus$ & $\oplus$ & $\odot$ & $\oplus$ & $\bigodot$ \\
\hline Zhang 2020 & $\oplus$ & $\oplus$ & $\oplus$ & $\odot$ & $\oplus$ & $\bigodot$ \\
\hline & & & & & & 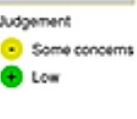 \\
\hline
\end{tabular}

Bias arising frem the randomization process

Biss due to deviations trom intended interventions

Biss due so missing oussomo cata
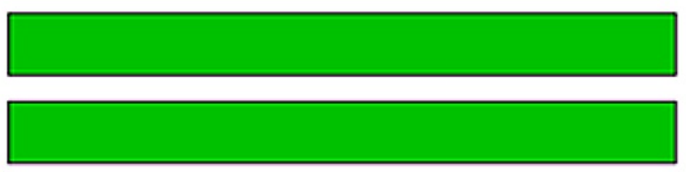

Bias in measurement of the outcome

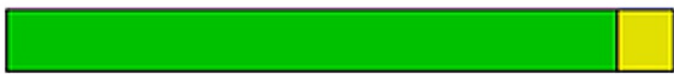

Bas in folestise of the reported result

Overal risk of blas
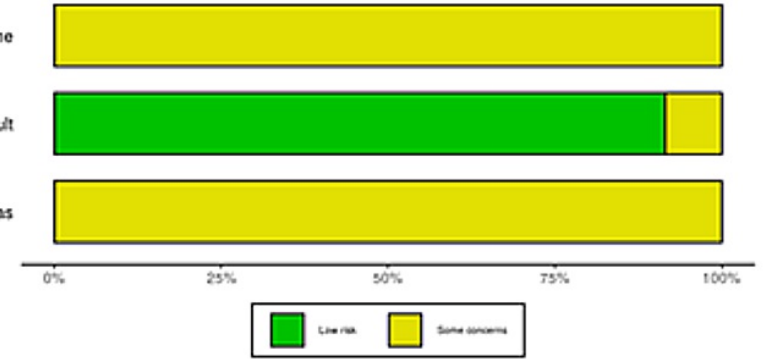

FIGURE 5: Risk-of-bias table and risk-of-bias graph for adverse events.

\section{Primary outcomes}

Ro Resection

Four studies reported complete resections $[9,27,28,30]$ and one reported R0 resections [8] from a total of 1,257 polyps. Although complete resection was often used as a synonym for R0 resection, each study had different definitions: one study defined R0 resection as en bloc resection with histologically confirmed negative lateral and vertical resection margins [8], one defined $\mathrm{R} 0$ resection as complete en bloc resection of a polyp with tumor-free lateral margins confirmed by negative biopsy [30], one defined it as pathological assessment of biopsy specimens from the resection margin [9], and another did not provide definitions. Therefore, we judged that a meta-analysis was not feasible, and the data were summarized narratively to rate the certainty in evidence using the GRADE approach (Tables 2, 3). Evidence derived from three RCTs showed that the proportion of R0 resection in the UEMR group was higher than that in the CEMR group $[8,27,28]$, while two reported no difference $[9,30]$. Our considered judgment was low certainty (rated down for very serious limitations in study design). 


\section{Cureus}

\begin{tabular}{|c|c|c|c|c|c|c|}
\hline \multirow{2}{*}{ Outcomes } & \multicolumn{2}{|c|}{ Anticipated absolute effects ${ }^{*}(95 \% \mathrm{Cl})$} & \multirow{2}{*}{$\begin{array}{l}\text { Relative } \\
\text { effect } \\
(95 \% \mathrm{Cl})\end{array}$} & \multirow{2}{*}{$\begin{array}{l}\text { Number } \\
\text { of polyps } \\
\text { (studies) }\end{array}$} & \multirow{2}{*}{$\begin{array}{l}\text { Certainty of } \\
\text { the evidence } \\
\text { (GRADE) }\end{array}$} & \multirow{2}{*}{ Comments } \\
\hline & Risk with CEMR & Risk with UEMR & & & & \\
\hline $\begin{array}{l}\text { R0 resection: } \\
\text { Assessed using a } \\
\text { variety of scales }\end{array}$ & \multicolumn{3}{|c|}{$\begin{array}{l}\text { Three studies showed that the } \mathrm{R} 0 \text { resection rate in the } \\
\text { UEMR group was higher than that in the CEMR group }\end{array}$} & $\begin{array}{l}1,257 \text { (5 } \\
\text { RCTs) }\end{array}$ & $\begin{array}{l}\oplus \oplus \bigcirc \bigcirc \\
\text { LOW }^{\text {a,b }}\end{array}$ & $\begin{array}{l}\text { UEMR may result in a } \\
\text { slight increase in Ro } \\
\text { resection }\end{array}$ \\
\hline En bloc resection & 582 per 1,000 & $\begin{array}{l}721 \text { per } 1,000(616 \\
\text { to } 806)\end{array}$ & $\begin{array}{l}\text { OR: } 1.84 \\
(1.14 \text { to } \\
2.96)\end{array}$ & $\begin{array}{l}1,374(6 \\
\text { RCTs) }\end{array}$ & $\begin{array}{l}\oplus \oplus \oplus О \\
\text { MODERATE }\end{array}$ & $\begin{array}{l}\text { UEMR likely results in a } \\
\text { large increase in en bloc } \\
\text { resection }\end{array}$ \\
\hline Procedure time & $\begin{array}{l}\text { The mean procedure } \\
\text { time was } 81 \text { to } 1,793 \\
\text { seconds }\end{array}$ & $\begin{array}{l}\text { MD } 64.5 \text { seconds } \\
\text { lower }(-122.9 \text { lower } \\
\text { to }-6.1 \text { lower) }\end{array}$ & - & $\begin{array}{l}1,257 \text { (5 } \\
\text { RCTs) }\end{array}$ & $\begin{array}{l}\oplus \oplus \oplus \bigcirc \\
\text { MODERATE }\end{array}$ & $\begin{array}{l}\text { UEMR likely results in a } \\
\text { decrease in procedure } \\
\text { time }\end{array}$ \\
\hline Adverse even & $\begin{array}{l}\text { There were } 0-17 \% \text { ad } \\
0-16 \% \text { adverse event }\end{array}$ & $\begin{array}{l}\text { verse events with the } \\
s \text { with the UEMR }\end{array}$ & EMR and & $\begin{array}{l}1,374(6 \\
\text { RCTs) }\end{array}$ & $\begin{array}{l}\oplus \oplus 00 \\
\text { Low }^{\text {a,c }}\end{array}$ & $\begin{array}{l}\text { UEMR may result in a } \\
\text { slight decrease in an } \\
\text { adverse event }\end{array}$ \\
\hline
\end{tabular}

\section{TABLE 2: Summary of findings: UEMR compared to CEMR for patients with colorectal polyp.}

${ }^{*}$ The risk in the intervention group (and its $95 \% \mathrm{Cl}$ ) is based on the assumed risk in the comparison group and the relative effect of the intervention (and its $95 \% \mathrm{Cl}$ ).

${ }^{a}$ Downgraded one level for very serious limitations in study design (allocation concealment which did not mask outcome assessment in all studies); ${ }^{b}$ Downgraded one level for very serious limitations in study design (surrogate outcomes are used in four studies; ${ }^{\mathrm{C}}$ Downgraded one level for very serious limitations in small sample size

GRADE Working Group grades of evidence. High certainty: We are very confident that the true effect lies close to that of the estimate of the effect. Moderate certainty: We are moderately confident in the effect estimate. The true effect is likely to be close to the estimate of the effect, but there is a possibility that it is substantially different. Low certainty: Our confidence in the effect estimate is limited. The true effect may be substantially different from the estimate of the effect. Very low certainty: We have very little confidence in the effect estimate. The true effect is likely to be substantially different from the estimate of effect.

CI: Confidence interval; OR: Odds ratio; MD: Mean Difference; UEMR: Underwater endoscopic mucosal resection; CEMR: Conventional endoscopic mucosal resection; GRADE: Grading of Recommendations Assessment, Development, and Evaluation

\begin{tabular}{|l|l|}
\hline $\begin{array}{l}\text { GRADE } \\
\text { domain }\end{array}$ & Judgment \\
\hline
\end{tabular}

Risk of bias

Inconsistency

Indirectness

Imprecision

bias

Downgraded one level for serious limitations in study design (allocation concealment which did not mask outcome assessment in all studies)

The patients, intervention, and comparators in the studies provide direct evidence to the clinical question at hand

Downgraded one level for serious limitations in study design (surrogate outcomes are used in four studies)

The total number of patients included in all the trials was 1,260 and the total number of events was 190. Although the number of events was relatively small, we assumed RRR as $50 \%$. Therefore, we judged the evidence was not imprecise

Publication We did not strongly suspect publication bias because both negative and positive trials were published, and the search for studies was comprehensive
Concerns

about

certainty

domains

Serious

Not serious

Serious

Not serious

Undetected

\section{TABLE 3: Rating the certainty in the evidence of R0 resection.}

GRADE: Grading of Recommendations Assessment, Development, and Evaluation; RRR: relative risk reduction 
Five studies reported procedure time $[8,9,27,28,30]$. We could include a total of 1,257 polyps in our analysis. UEMR likely resulted in a large reduction in procedure time, although there was significant heterogeneity ( $\mathrm{MD}=-64.5$ seconds; $95 \% \mathrm{CI}=-122.9$ to -6.1 seconds; $\mathrm{I}^{2}=86 \%$; moderate certainty of the evidence) (Figure 6).

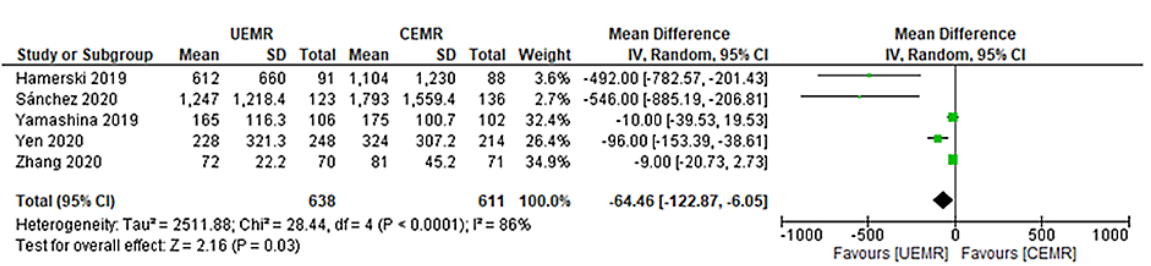

UEMR: Underwater endoscopic mucosal resection; CEMR: Conventional endoscopic mucosal resection; SD: standard deviation; CI: confidence interval.

FIGURE 6: Forest plot comparing procedure time between UEMR and CEMR.

\section{Secondary outcomes}

En Bloc Resection

All six studies reported en bloc resection [8,9,27-30]. We could include a total of 1,374 polyps in our analysis. UEMR likely resulted in a large increase in the proportion of en bloc resection (odds ratio $=1.84 ; 95 \% \mathrm{CI}=$ 1.14 to $2.96 ; \mathrm{I}^{2}=59 \%$; moderate certainty of the evidence) (Figure 7).

\begin{tabular}{|c|c|c|c|c|c|c|c|c|}
\hline Study or Subgroup & \multicolumn{2}{|c|}{ CEMR } & \multicolumn{2}{|c|}{ UEMR } & \multicolumn{2}{|c|}{$\begin{array}{c}\text { Odds Ratio } \\
\text { Weight } \mathrm{M} \cdot \mathrm{H}, \text { Random, } 95 \% \mathrm{Cl}\end{array}$} & \multicolumn{2}{|c|}{$\begin{array}{l}\text { Odds Ratio } \\
\text { M.H, Random, } 95 \% \mathrm{Cl}\end{array}$} \\
\hline Hamerski 2019 & 68 & 88 & 41 & 91 & $19.0 \%$ & $4.15[2.17,7.92]$ & & \\
\hline Nagl 2020 & 48 & 59 & 42 & 58 & $14.8 \%$ & $1.66[0.69,3.98]$ & - & \\
\hline Sánchez 2020 & 113 & 141 & 94 & 126 & $20.5 \%$ & $1.37[0.77,2.44]$ & & \\
\hline Yamashina 2019 & 26 & 102 & 12 & 106 & $17.0 \%$ & $2.68[1.27,5.66]$ & & \\
\hline Yen 2020 & 21 & 214 & 25 & 248 & $19.7 \%$ & $0.97[0.53,1.79]$ & - & - \\
\hline Zhang 2020 & 6 & 71 & 4 & 70 & $9.1 \%$ & $1.52[0.41,5.65]$ & & \\
\hline Total $(95 \% \mathrm{Cl})$ & & 675 & & 699 & $100.0 \%$ & $1.84[1.14,2.96]$ & & \\
\hline Total events & 282 & & 218 & & & & & \\
\hline $\begin{array}{l}\text { Heterogeneity. Tauz= } \\
\text { Test for overall effect }\end{array}$ & $\begin{array}{l}0.20 ; \mathrm{Chi}^{2} \\
\mathrm{Z}=2.51(\mathrm{P}\end{array}$ & $\begin{array}{l}=12.3 \\
P=0.0\end{array}$ & 33, $d f=5$ & $P=0$. & 3); $1^{2}=$ & & 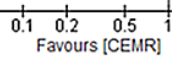 & 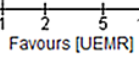 \\
\hline
\end{tabular}

UEMR: Underwater endoscopic mucosal resection; CEMR: Conventional endoscopic mucosal resection; SD: standard deviation; CI: confidence interval.

\section{FIGURE 7: Forest plot comparing en bloc resection rate between UEMR and CEMR.}

All Adverse Events

All six studies reported all adverse events and included a total of 1,374 polyps [8,9,27-30]. One study reported no adverse events [9], and five studies reported adverse events, and almost all of them were delayed bleeding $[8,27-30]$ (Table 2). There were $0-17 \%$ adverse events with CEMR and $0-16 \%$ adverse events with UEMR.

Subgroup Analysis

Subgroup analyses indicated that procedure time was significantly shorter for lesions measuring $>20 \mathrm{~mm}$ versus $\leqslant 20 \mathrm{~mm}\left(\mathrm{I}^{2}=94.5 \%\right.$; $\left.<0.0001 ; \mathrm{chi}^{2}=18.3\right)$, and the procedure time for UEMR was significantly shorter than for CEMR for lesions $>20 \mathrm{~mm}\left(\mathrm{MD}=-514.9\right.$ seconds; $95 \% \mathrm{CI}=-735.5$ to -294.2 seconds; $\mathrm{I}^{2}=0 \%$ ) but not for lesions $\leqslant 20 \mathrm{~mm}$ ( $\mathrm{MD}=-27.2$ seconds; $95 \% \mathrm{CI}=-62.0$ to 7.5 seconds; $\mathrm{I}^{2}=76 \%$ ). However, there was no significant difference in the rates of en bloc resection between lesions $>20 \mathrm{~mm}$ and $\leqslant 20 \mathrm{~mm}\left(\mathrm{I}^{2}=0 \%\right.$; $\mathrm{p}=0.53 ; \mathrm{chi}^{2}=0.39 ; \mathrm{I}^{2}=0 \%$ ). UEMR showed a significant increase in the rate of en bloc resection for lesions 
$>20 \mathrm{~mm}$ compared with CEMR (odds ratio $=2.13 ; 95 \% \mathrm{CI}=1.03$ to $4.38 ; \mathrm{I}^{2}=70 \%$ ) but not for lesions $\leqslant 20 \mathrm{~mm}$ (odds ratio $=1.55 ; 95 \% \mathrm{CI}=0.77$ to $3.12 ; \mathrm{I}^{2}=53 \%$ ) (Figures 8,9 ).

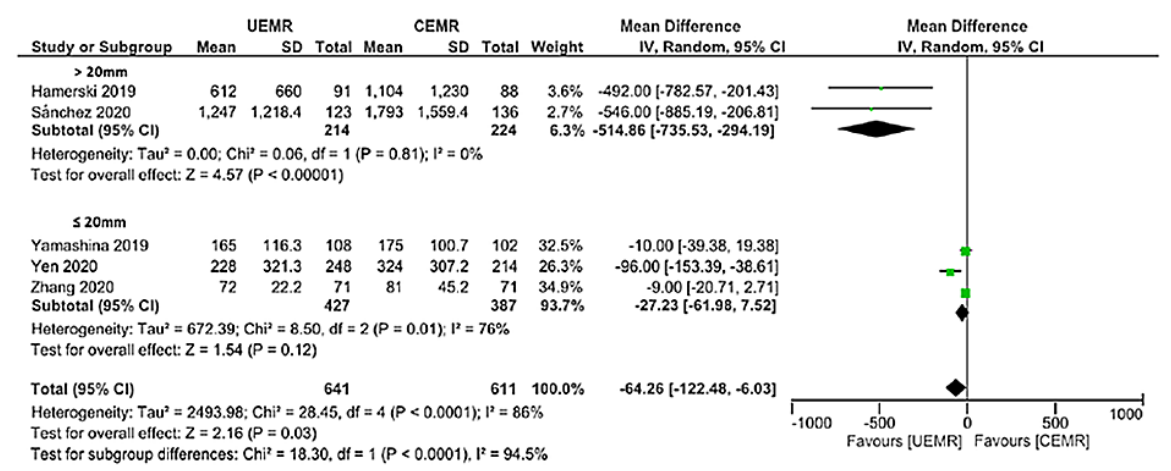

UEMR: Underwater endoscopic mucosal resection; CEMR: Conventional endoscopic mucosal resection; SD: standard deviation; CI: confidence interval.

FIGURE 8: Forest plot comparing procedure time between UEMR and CEMR on the size of the lesion ( $\leq 20$ or $>20 \mathrm{~mm}$ ).

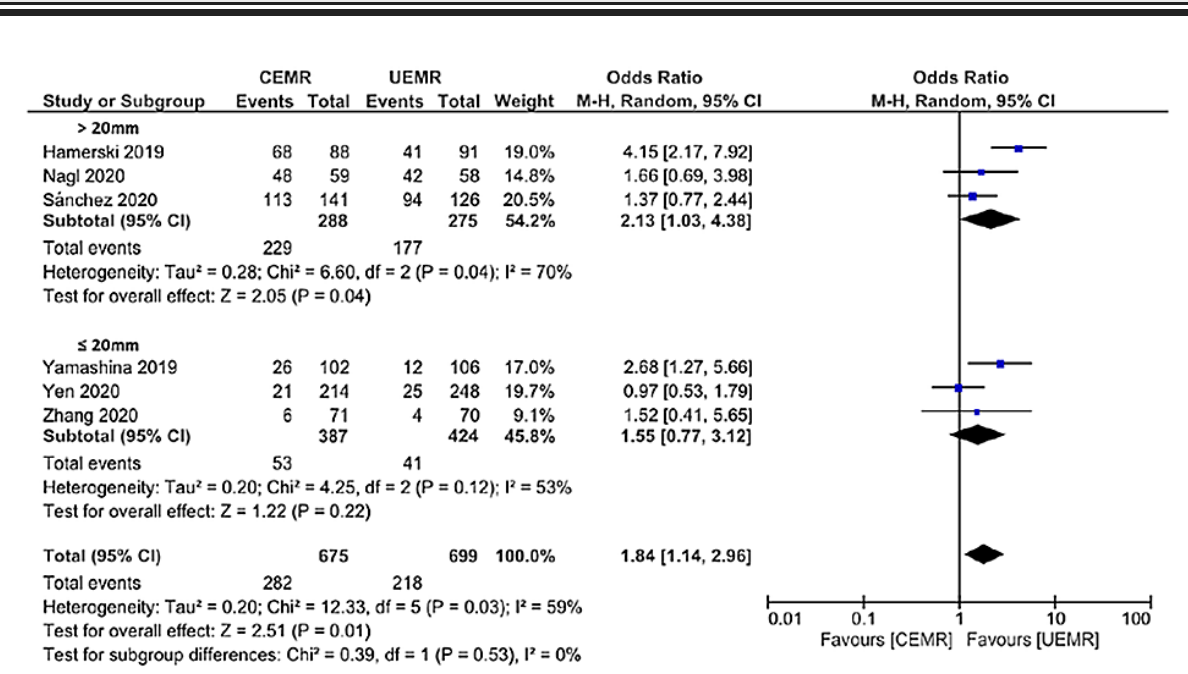

UEMR: Underwater endoscopic mucosal resection; CEMR: Conventional endoscopic mucosal resection; SD: standard deviation; CI: confidence interval.

FIGURE 9: Forest plot comparing en bloc resection between UEMR and CEMR on the size of the lesion ( $\leq 20$ or $>20 \mathrm{~mm}$ ).

\section{Discussion}

This review of six RCTs and 1,374 polyps found that UEMR might have better efficacy than CEMR in endoscopic resection for nonpedunculated colorectal polyps. Although several systematic reviews and metaanalyses have already been published and have reported UEMR as effective for the resection of flat colorectal polyps, their data are insufficient because the results were derived from only a single-arm UEMR trial [10] or a mixture of cohort trials and RCTs [11-14].

Tziatzios et al. showed that the rate of en bloc resection was significantly higher in the UEMR group in their meta-analysis; however, there was no significant difference in the complete resection rate between the UEMR and CEMR groups, and these results were divergent [15]. We judged that a meta-analysis was not feasible and summarized the data narratively for our primary outcome, the proportion of R0 resection. The reasons for doing so were that each study had different definitions of R0 resection (we regarded complete resection as a synonym) with the major difference related to whether the negative resection margin was confirmed endoscopically or histologically. Another difference was whether histological confirmation was 
achieved using a complete en bloc resected specimen, piecemeal resected specimen, or biopsy specimen near the polyp. Of course, histological assessment of en bloc resected specimens is the most desirable method for confirmation. However, as the polyp size increases, it becomes more difficult to obtain complete en bloc resection, and multiple cases are needed to conduct RCTs. In the present study, the UEMR group had higher efficacy than the CEMR group in three RCTs [8,27,28] while another two showed no difference [9,30]. These two studies mainly targeted small polyps less than $10 \mathrm{~mm}$ in size. Our subgroup analysis also showed that UEMR was better than CEMR regarding the proportion of en bloc resection rate and procedure time for colorectal polyps $>20 \mathrm{~mm}$. The proportion of R0 resection is substantial for small polyps, with little benefit being derived from water immersion. If the polyp size is larger than $10 \mathrm{~mm}$, however, R0 resection becomes less successful [20]. Because these cases may result in local recurrence after piecemeal resection, UEMR is considered clinically important for polyps larger than $10 \mathrm{~mm}$.

In this study, we found that UEMR might be associated with a higher proportion of en bloc resection and shorter procedure time than CEMR. In line with four previous systematic reviews and meta-analyses demonstrating that UEMR was associated with a statistically significantly higher proportion of en bloc resection than CEMR [11-14], our results also showed that UEMR likely results in a large increase in en bloc resection. UEMR was first reported by Binmoeller et al. [5], and water immersion, the main point of UEMR, is thought to have several advantages that support our results, namely, the colorectal wall extension force is decreased, which is accompanied by increased mucosal shrink and flotation upward into the lumen. This results in flat mucosal lesions becoming polypoid, which makes snaring easier and permits eventual total en bloc resection.

Although previous systematic reviews and meta-analyses have not clearly analyzed the comparison of the procedure time for UEMR versus CEMR, our results showed that UEMR likely results in a large reduction in procedure time. Submucosal injection requires complicated maneuvers and several steps before resection, such as good positional control of the colonoscope to the polyp, insertion of the injection needle into the colonoscope, correct injection into the submucosa, creation of sufficient submucosal fluid elevation, and removal of the injection needle, which are time-consuming. In contrast, UEMR requires only deflation of $\mathrm{air} / \mathrm{CO}_{2}$ and injection of water into the lumen. In addition to the advantages already mentioned, water

immersion minimizes luminal distension and flexure angulation, which improves endoscopic maneuverability. For these reasons, UEMR is likely to save time.

As CEMR has already been broadly generalized for resecting colorectal polyps in daily clinical settings, it should also be possible to generalize UEMR. Submucosal injection can create a cushion in the submucosal layer and enhance the safety of hot snare polypectomy by reducing deep thermal injury [2], which is thought to be a potential risk factor for adverse events. Therefore, considering submucosal injection prior to hot snare polypectomy is recommended [20], and CEMR has become very popular because of this safety aspect. However, Binmoeller et al. demonstrated with endosonographic observation that with underwater endoscopy, deflation of air $/ \mathrm{CO}_{2}$ and immersion in water ensure safety by separating the mucosa and submucosa away from the muscularis propria [5]. Submucosal injection requires a considerable level of proficiency to avoid misguidance into the muscularis propria, poor elevation for snaring, and formation of a hematoma. The underwater procedure, however, is relatively easy to learn because it requires only deflation of air/ $\mathrm{CO}_{2}$ and injection of water into the lumen [5]. Therefore, UEMR is potentially easily generalizable to the daily clinical setting.

Several limitations of this study must be mentioned. First, four of the six studies, three of which were in abstract form, showed a high risk of bias in the selection of the reported results owing to the lack of a preregistered protocol or no pre-specified outcomes. Nevertheless, we asked the original authors for protocol and prespecified outcomes as much as possible and evaluated the data to the maximum extent possible. Second, the recurrence rate was inadequately evaluated in the six eligible RCTs. Two of the six studies did not evaluate long-term follow-up and recurrence, and the follow-up rates of the other four studies were not high enough. Therefore, a well-designed follow-up study of local recurrence after UEMR and CEMR is warranted. Third, the participating endoscopists were unblinded to the group allocations. Endoscopists perform UEMR or CEMR according to the allocation results; therefore, this problem is unavoidable in RCTs.

\section{Conclusions}

This systematic review and meta-analysis showed that UEMR might have higher efficacy than CEMR in endoscopic resection of nonpedunculated colorectal polyps, with a probable large reduction in procedure time and increased proportion of en bloc resection. The findings suggest that endoscopists might preferably perform UEMR rather than CEMR for nonpedunculated colorectal polyps. However, meta-analysis for R0 resection was not feasible in this study; therefore, further high-quality RCTs are needed.

\section{Appendices}




\section{Cureus}

\begin{tabular}{|c|c|c|c|c|}
\hline Section/topic & \# & Checklist item & $\begin{array}{l}\text { Reported } \\
\text { on page } \\
\text { number }\end{array}$ & \\
\hline \multicolumn{5}{|l|}{ TITLE } \\
\hline Title & 1 & Identify the report as a systematic review, meta-analysis, or both & 1 & \\
\hline ABSTRACT & & & & 3,4 \\
\hline $\begin{array}{l}\text { Structured } \\
\text { summary }\end{array}$ & 2 & $\begin{array}{l}\text { Provide a structured summary including, as applicable: background; objectives; data sources; } \\
\text { study eligibility criteria, participants, and interventions; study appraisal and synthesis methods; } \\
\text { results; limitations; conclusions and implications of key findings; systematic review registration } \\
\text { number }\end{array}$ & 3,4 & \\
\hline \multicolumn{5}{|l|}{ INTRODUCTION } \\
\hline Rationale & 3 & Describe the rationale for the review in the context of what is already known & 5 & \\
\hline Objectives & 4 & $\begin{array}{l}\text { Provide an explicit statement of questions being addressed with reference to participants, } \\
\text { interventions, comparisons, outcomes, and study design (PICOS) }\end{array}$ & 5 & \\
\hline \multicolumn{5}{|l|}{ METHODS } \\
\hline $\begin{array}{l}\text { Protocol and } \\
\text { registration }\end{array}$ & 5 & $\begin{array}{l}\text { Indicate if a review protocol exists, if and where it can be accessed (e.g., Web address), and, if } \\
\text { available, provide registration information including registration number }\end{array}$ & 6 & \\
\hline $\begin{array}{l}\text { Eligibility } \\
\text { criteria }\end{array}$ & 6 & $\begin{array}{l}\text { Specify study characteristics (e.g., PICOS, length of follow-up) and report characteristics (e.g., } \\
\text { years considered, language, publication status) used as criteria for eligibility, giving rationale }\end{array}$ & 6,7 & \\
\hline $\begin{array}{l}\text { Information } \\
\text { sources }\end{array}$ & 7 & $\begin{array}{l}\text { Describe all information sources (e.g., databases with dates of coverage, contact with study } \\
\text { authors to identify additional studies) in the search and date last searched }\end{array}$ & 8 & \\
\hline Search & 8 & $\begin{array}{l}\text { Present full electronic search strategy for at least one database, including any limits used, such } \\
\text { that it could be repeated }\end{array}$ & $\begin{array}{l}8 \\
\text { Appendix } \\
2\end{array}$ & \\
\hline $\begin{array}{l}\text { Study } \\
\text { selection }\end{array}$ & 9 & $\begin{array}{l}\text { State the process for selecting studies (i.e., screening, eligibility, included in the systematic } \\
\text { review, and, if applicable, included in the meta-analysis) }\end{array}$ & 7 & \\
\hline $\begin{array}{l}\text { Data } \\
\text { collection } \\
\text { process }\end{array}$ & 10 & $\begin{array}{l}\text { Describe the method of data extraction from reports (e.g., piloted forms, independently, in } \\
\text { duplicate) and any processes for obtaining and confirming data from investigators }\end{array}$ & 7,8 & \\
\hline Data items & 11 & $\begin{array}{l}\text { List and define all variables for which data were sought (e.g., PICOS, funding sources) and any } \\
\text { assumptions and simplifications made }\end{array}$ & 9,10 & \\
\hline $\begin{array}{l}\text { Risk of bias in } \\
\text { individual } \\
\text { studies }\end{array}$ & 12 & $\begin{array}{l}\text { Describe methods used for assessing the risk of bias of individual studies (including } \\
\text { specification of whether this was done at the study or outcome level), and how this information } \\
\text { is to be used in any data synthesis }\end{array}$ & 10,11 & \\
\hline $\begin{array}{l}\text { Summary } \\
\text { measures }\end{array}$ & 13 & State the principal summary measures (e.g., risk ratio, difference in means) & 10 & \\
\hline $\begin{array}{l}\text { Synthesis of } \\
\text { results }\end{array}$ & 14 & $\begin{array}{l}\text { Describe the methods of handling data and combining results of studies, if done, including } \\
\left.\text { measures of consistency (e.g., }\left.\right|^{2}\right) \text { for each meta-analysis }\end{array}$ & 11 & \\
\hline
\end{tabular}

TABLE 4: PRISMA 2009 checklist.

PRISMA: Preferred Reporting Items for Systematic Reviews and Meta-Analyses

\section{Additional Information Disclosures}

Conflicts of interest: In compliance with the ICMJE uniform disclosure form, all authors declare the following: Payment/services info: All authors have declared that no financial support was received from any organization for the submitted work. Financial relationships: All authors have declared that they have no financial relationships at present or within the previous three years with any organizations that might 
have an interest in the submitted work. Other relationships: All authors have declared that there are no other relationships or activities that could appear to have influenced the submitted work.

\section{Acknowledgements}

This work was supported by the Systematic Review Workshop Peer Support Group (SRWS-PSG). The authors would like to thank Dr. Marco A. Alvarez-Gonzalez and Dr. Kenneth Binmoeller for providing us with unpublished details of studies for the review. We thank Hugh McGonigle, from Edanz (https://jp.edanz.com/ac), for editing a draft of the manuscript.

\section{References}

1. Sung H, Ferlay J, Siegel RL, Laversanne M, Soerjomataram I, Jemal A, Bray F: Global Cancer Statistics 2020: GLOBOCAN estimates of incidence and mortality worldwide for 36 cancers in 185 countries. CA Cancer J Clin. 2021, 71:209-49. 10.3322/caac.21660

2. Deyhle P, Largiadèr F, Jenny S, Fumagalli I: A method for endoscopic electroresection of sessile colonic polyps. Endoscopy. 1973, 5:38-40. 10.1055/s-0028-1098209

3. Hurlstone DP, Sanders DS, Cross SS, et al.: Colonoscopic resection of lateral spreading tumours: a prospective analysis of endoscopic mucosal resection. Gut. 2004, 53:1334-9. 10.1136/gut.2003.036913

4. Saito Y, Fukuzawa M, Matsuda T, et al.: Clinical outcome of endoscopic submucosal dissection versus endoscopic mucosal resection of large colorectal tumors as determined by curative resection. Surg Endosc. 2010, 24:343-52. 10.1007/s00464-009-0562-8

5. Binmoeller KF, Weilert F, Shah J, Bhat Y, Kane S: "Underwater" EMR without submucosal injection for large sessile colorectal polyps (with video). Gastrointest Endosc. 2012, 75:1086-91. 10.1016/j.gie.2011.12.022

6. Kim HG, Thosani N, Banerjee S, Chen A, Friedland S: Underwater endoscopic mucosal resection for recurrences after previous piecemeal resection of colorectal polyps (with video). Gastrointest Endosc. 2014, 80:1094-102. 10.1016/j.gie.2014.05.318

7. Yamashina T, Tumura T, Maruo T, et al.: Underwater endoscopic mucosal resection: a new endoscopic method for resection of rectal neuroendocrine tumor grade 1 (carcinoid) $\leqslant 10 \mathrm{~mm}$ in diameter. Endosc Int Open. 2018, 6:E111-4. 10.1055/s-0043-123467

8. Yamashina T, Uedo N, Akasaka T, et al.: Comparison of underwater vs conventional endoscopic mucosal resection of intermediate-size colorectal polyps. Gastroenterology. 2019, 157:451-61.e2. 10.1053/j.gastro.2019.04.005

9. Yen AW, Leung JW, Wilson MD, Leung FW: Underwater versus conventional endoscopic resection of nondiminutive nonpedunculated colorectal lesions: a prospective randomized controlled trial (with video). Gastrointest Endosc. 2020, 91:643-54.e2. 10.1016/j.gie.2019.09.039

10. Spadaccini M, Fuccio L, Lamonaca L, et al.: Underwater EMR for colorectal lesions: a systematic review with meta-analysis (with video). Gastrointest Endosc. 2019, 89:1109-16.e4. 10.1016/j.gie.2018.10.023

11. Li P, Ma B, Gong S, Zhang X, Li W: Underwater endoscopic mucosal resection for colorectal lesions: a metaanalysis. Surg Endosc. 2021, 35:3003-13. 10.1007/s00464-020-07745-8

12. Ni DQ, Lu YP, Liu XQ, Gao LY, Huang X: Underwater vs conventional endoscopic mucosal resection in treatment of colorectal polyps: a meta-analysis. World J Clin Cases. 2020, 8:4826-37. 10.12998/wjcc.v8.i20.4826

13. Choi AY, Moosvi Z, Shah S, Roccato MK, Wang AY, Hamerski CM, Samarasena JB: Underwater versus conventional EMR for colorectal polyps: systematic review and meta-analysis. Gastrointest Endosc. 2021, 93:378-89. 10.1016/j.gie.2020.10.009

14. Kamal F, Khan MA, Lee-Smith W, et al.: Underwater vs conventional endoscopic mucosal resection in the management of colorectal polyps: a systematic review and meta-analysis. Endosc Int Open. 2020, 8:E126472. 10.1055/a-1214-5692

15. Tziatzios G, Gkolfakis P, Triantafyllou K, et al.: Higher rate of en bloc resection with underwater than conventional endoscopic mucosal resection: a meta-analysis. Dig Liver Dis. 2021, 53:958-64. 10.1016/j.dld.2021.05.001

16. Moher D, Liberati A, Tetzlaff J, Altman DG: Preferred reporting items for systematic reviews and metaanalyses: the PRISMA statement. Int J Surg. 2010, 8:336-41. 10.1016/j.ijsu.2010.02.007

17. Higgins JP, Green S: Cochrane handbook for systematic reviews of interventions | Cochrane Training . John Wiley \& Sons Ltd., Sussex, UK; 2019. 10.1002/9780470712184

18. Efficacy of underwater EMR for non-pedunculated colorectal lesions: a systematic review and meta-analysis protocol. (2020). Accessed: Aug 24, 2020: https://www.protocols.io/search?q=bj5rkq56.

19. The Paris endoscopic classification of superficial neoplastic lesions: esophagus, stomach, and colon: November 30 to December 1, 2002. Gastrointest Endosc. 2003, 58:S3-43. 10.1016/s0016-5107(03)02159-X

20. Ferlitsch M, Moss A, Hassan C, et al.: Colorectal polypectomy and endoscopic mucosal resection (EMR): European Society of Gastrointestinal Endoscopy (ESGE) Clinical Guideline. Endoscopy. 2017, 49:270-97. 10.1055/s-0043-102569

21. Tanaka S, Saitoh Y, Matsuda T, et al.: Evidence-based clinical practice guidelines for management of colorectal polyps. J Gastroenterol. 2015, 50:252-60. 10.1007/s00535-014-1021-4

22. Kaltenbach T, Anderson JC, Burke CA, et al.: Endoscopic removal of colorectal lesions-recommendations by the US Multi-Society Task Force on colorectal cancer. Gastroenterology. 2020, 158:1095-129. 10.1053/j.gastro.2019.12.018

23. Hashiguchi Y, Muro K, Saito Y, et al.: Japanese Society for Cancer of the Colon and Rectum (JSCCR) guidelines 2019 for the treatment of colorectal cancer. Int J Clin Oncol. 2020, 25:1-42. 10.1007/s10147-019$01485-\mathrm{Z}$

24. Sterne JA, Savović J, Page MJ, et al.: RoB 2: a revised tool for assessing risk of bias in randomised trials . BMJ. 2019, 366:14898. 10.1136/bmj.14898 


\section{Cureus}

25. Campbell M, McKenzie JE, Sowden A, et al.: Synthesis without meta-analysis (SWiM) in systematic reviews: reporting guideline. BMJ. 2020, 368:16890. 10.1136/bmj.16890

26. Murad MH, Mustafa RA, Schünemann HJ, Sultan S, Santesso N: Rating the certainty in evidence in the absence of a single estimate of effect. Evid Based Med. 2017, 22:85-7. 10.1136/ebmed-2017-110668

27. Hamerski CM, Wang AY, Amato A, et al.: Injection-assisted versus underwater endoscopic mucosal resection without injection for the treatment of colorectal laterally spreading tumors: interim analysis of an international multicenter randomized controlled trial. Gastrointest Endosc. 2018, 87:AB55-6. 10.1016/j.gie.2018.04.033

28. Rodríguez Sánchez J, Coto Ugarte D, Koeklin H, et al.: Efficacy of underwater endoscopic mucosal resection for the treatment of large complex colorectal lesions: a randomized and multicenter control trial. Endoscopy. 2020, 52:S111. 10.1055/s-0040-1704342

29. Nagl S, Ebigbo A, Braun G, et al.: Underwater versus conventional endoscopic mucosal rejection of large sessile or flat sessile or flat colonic polyps: preliminary results of a prospective randomized controlled trial. Endoscopy. 2020, 52(S 01):S6. 10.1055/s-0040-1704026

30. Zhang Z, Xia Y, Cui H, et al.: Underwater versus conventional endoscopic mucosal resection for small size non-pedunculated colorectal polyps: a randomized controlled trial: (UEMR vs. CEMR for small size nonpedunculated colorectal polyps). BMC Gastroenterol. 2020, 20:311. 10.1186/s12876-020-01457-y 